\title{
CORRECTIONS
}

\section{Lessons from the fatal French study BIA-10-2474}

In this editorial (BMJ 2016;353:i2727, doi:10.1136/bmj.i2727) the second paragraph of the section titled "The drug" contains an error. The dose increase specified in parenthesis for the multiple ascending doses substudy should read "from $20 \mathrm{mg} /$ day in the fourth series to $50 \mathrm{mg} /$ day in the fifth series," not "from $20 \mathrm{mg}$ on day 4 to $50 \mathrm{mg}$ on day 5." 